\title{
Screening and Identification of a Quercetin-Producing Bacterium Paenibacillus glucanolyticu D3
}

\author{
Zhitang Lu (Corresponding author) \\ College of Life Sciences \\ Hebei University and Key Laboratory of Microbial Diversity Research and Application of Hebei Province \\ Baoding 071002, China
}

Tel: 86-312-507-9696_E-mail: lzt325@sina.com

\author{
Yating Zhang \\ College of Life Sciences
}

Hebei University and Key Laboratory of Microbial Diversity Research and Application of Hebei Province

Baoding 071002, China

Tel: 86-312-507-9696Ｅ-mail: zhangyating00001@126.com

Nan Shi

College of Life Sciences

Hebei University and Key Laboratory of Microbial Diversity Research and Application of Hebei Province

Baoding 071002, China

Tel: 86-312-507-9696Ｅ-mail: eshishi@126.com

Received: January 3, 2012

Accepted: February 6, 2012 Published: March 1, 2012

doi:10.5539/enrr.v2n1p106

URL: http://dx.doi.org/10.5539/enrr.v2n1p106

\begin{abstract}
Quercetin is a kind of flavonoid, which has outstanding drug action. To develop the production technology of quercetin by microorganism, strains that can transform rutin to quercetin were isolated from soil, screened by TLC and re-screened by HPLC. As a result, strain D3 with the highest transformation ability was obtained. The hydrolyze enzyme was intracellular enzyme and transformation rate was $41.6 \%$ within a system comprising of $50 \mathrm{mg}$ wet cells in $4 \mathrm{~mL}$ of $0.1 \%$ rutin solution and transformed for $72 \mathrm{hr}$ at $25^{\circ} \mathrm{C}$. Strain D3 was identified as Paenibacillus glucanolyticus by integrating the $16 \mathrm{~S}$ rDNA phylogeny analysis result and a variety of morphological, physiological and biochemical features.
\end{abstract}

Keywords: Rutin, Quercetin, Transformation, Identification

\section{Introduction}

Flos Sophorae is the dried flowers and buds of Chinese scholartree Sophora japonica L., which originates in the north of China, and widely distribute in the south and southwest areas nowadays. Traditional Chinese medicine considers that Flos Sophorae has a number of drug effects, such as, cooling blood, hemostasis, clearing away heat and reducing fire and so on. The most important use of it is treating hematochezia, metrorrhagia and metrostaxis and liverfire. Recently researches indicate that Flos Sophorae contains abundant physiological active components and nutrients, and shows remarkable curative effect as natural medicine and high quality feed in clinic. Moreover the water extract of Flos Sophorae has significant effect on anti-bacteria, anti-inflammatory, antiviral, etc (Sun et al., 2009).

The major effective components of Flos sophorae are flavonoids composed of large quantity of rutin (24.46\%, $\mathrm{w} / \mathrm{w})$ and little amount of quercetin (1.41\%, w/w) (Cheng et al., 2004) (Fig.1). Both rutin and quercetin are medicines and have many physiological activities. They have similar activities in eliminating free-radicals, 
antioxidative activities and protective effect in the hypoxia/hypoglycemia model of bacteria precipitation, antioxidative activities in vitro and anti-lipid peroxidation, but quercitin act preponderant function than rutin (Jin et al., 2007; Jin et al., 2009; Su et al., 2002; Jiang et al., 2007). At present, only little part of Flos sophoraes are used for rutin extraction, most of them are wasted.

It had been reported that rutin could be degraded by microorganisms, such as Aspergihs flavus (Krishnamurty et al., 1970), Penicillum rugulosum (Narikawa et al., 2000) and Thermoactinomyces vulgaris (Yang et al., 2009), and so on. These microorganisms produced $\beta$-glucosidases which converted rutin (quercetin-3-O- $\beta$-D-glucose- $\alpha$-L-rhamnose) to quercetin. But till now quercetin is obtained mainly by acid hydrolyzed in modern industry production, and large quantity of acidic waste water is produced. The reason is due to the low activity of the rutin degrade enzymes and the limited solubility of the rutin, which lead to a very low productivity. Hence, it is necessary to develop a simple, economical, environment friendly and efficient method for quercetin production.

This study aimed to screen potential bacteria that can produce novel type of rutinosidase and transform rutin to quercetin with high efficiency from soil samples collected in accumulation area of Chinese scholartree. If this procedure comes to reality in industry, it will not only solve the waste of Flos sophorae as resources and improves its additional value, but also can reduce the environmental pollution caused by the quercetin acid hydrolysis technology.

\section{Materials and Methods}

\subsection{Samples}

Soil samples were collected from decomposed leaves accumulation area of Chinese scholartree in Hebei University campus.

\subsection{Medium and reagent}

Beef extract-peptone medium (pH7.0) was used for bacteria isolation and cultivation. Rutin (AR), standard reagents of rutin and quercetin were purchased from Chengdu Must Bio-Technology Co., Ltd.

\subsection{Isolation of quercetin-producing bacteria}

$10 \mathrm{~g}$ soil sample was suspended in sterile water ( $\mathrm{pH} 7.0$ ) with 20 glass beades and oscillated at $25^{\circ} \mathrm{C}$ for 25 min. Quercetin-producing bacteria were isolated using serial dilution method by spread suitable dilutions on Beef extract-peptone medium supplemented with $0.1 \%(\mathrm{w} / \mathrm{w})$ rutin. After incubate for $1 \mathrm{~d}$ at $25^{\circ} \mathrm{C}$, bacterium-like colonies were inoculated, and those colonies with deep-colored hydrolysis zones after sprayed $1.0 \%$ $\mathrm{AlCl}_{3}$-ethanol solution were selected for screening.

\subsection{Screening techniques}

\subsubsection{Preliminary screening}

Thin layer chromatography (TLC) method was used to screen the active strains. The isolate was cultured in $4 \mathrm{~mL}$ of Beef extract-peptone broth (pH7.0) in $15 \times 150 \mathrm{~mm}$ test tubes. Cultivation was conducted at $25^{\circ} \mathrm{C}$ in a thermostat shaker by shaking at $280 \mathrm{r} / \mathrm{min}$ for $1 \mathrm{~d}$. The liquid culture was centrifuged at $4500 \mathrm{r} / \mathrm{min}$ for $5 \mathrm{~min}$ at room temperature $\left(24^{\circ} \mathrm{C} \pm 1^{\circ} \mathrm{C}\right)$ to separate the cell and the supernatant. The cell pellet was washed with $2 \mathrm{~mL}$ of $0.1 \mathrm{~mol} / \mathrm{L}$ phosphate buffer ( $\mathrm{pH} 7.0$ ) and centrifuged again to harvest the biomass. Then the bacteria biomass was suspended with $4 \mathrm{~mL}$ of phosphate buffer (pH7.0). $4 \mathrm{~mL}$ of cell suspension and the fermentation supernatant were supplemented with $0.1 \%$ rutin and react for $3 \mathrm{~d}$ by shaking at $280 \mathrm{r} / \mathrm{min}$ and $25^{\circ} \mathrm{C}$, repetitively. At last, TLC analysis was performed to check the activity of transforming rutin to quercetin.

The TLC analysis was carried out by loaded the sample on polyamide film and developed with $75 \%$ microemulsion consisted of SDS/n-butyl alcohol/n-heptane/water, 0.27: 0.63: 0.10: $36(\mathrm{~m} / \mathrm{m})$ (Kang et al., 2000). The developed polyamide film was stained by spraying $1 \% \mathrm{AlCl}_{3}$-ethanol solution and dried under room temperature. TLC polyamide film were scanned and analyzed by UN-scan-it gel scanning software (SIM International Group Co. LTD. U.S.A.).

\subsubsection{Secondary screening}

After preliminary screening, High Performance Liquid Chromatography (HPLC) was used to re-screen the active strains by comparing the retained time of converted products with quercetin standard. The chromatography conditions were as following: separated by pre-packed Hypersil ODS (C18) column (250×4.6mm, Elite, China) column at $24^{\circ} \mathrm{C} \pm 1{ }^{\circ} \mathrm{C}$ and detected by UV detector at $360 \mathrm{~nm}$, the mobile phase composed of acetonitrile: 
methanol: water: phosphoric acid $(100: 10: 340: 0.3, \mathrm{v} / \mathrm{v})$ was used as the eluant at a flow rate of $1.0 \mathrm{~mL} / \mathrm{min}$, sample volume was $20 \mu \mathrm{L}$ (Jia et al., 2008).

The HPLC sample was prepared by concentrating $4 \mathrm{~mL}$ of converted liquid to $200 \mu \mathrm{L}$ at $40^{\circ} \mathrm{C}$, supplemented with $300 \mu \mathrm{L}$ of methanol, centrifuged at $4500 \mathrm{r} / \mathrm{min}$ for $5 \mathrm{~min}$ to wipe off the precipitation, and made up to $500 \mu \mathrm{L}$.

\subsection{Transformation rate determination}

Diluted standards solution of rutin and quercetin were prepared by using $4.90 \mathrm{mg}$ rutin and $5.88 \mathrm{mg}$ quercetin and they were used for preparation of different working standards using methanol.

$20 \mu \mathrm{L}$ of the standard solution of each concentration (rutin solution: $0.272 \mu \mathrm{g} / \mathrm{mL}, 0.544 \mu \mathrm{g} / \mathrm{mL}, 1.089 \mu \mathrm{g} / \mathrm{mL}$, $2.178 \mu \mathrm{g} / \mathrm{mL}, \quad 4.356 \mu \mathrm{g} / \mathrm{mL}$; quercetin solution: $0.327 \mu \mathrm{g} / \mathrm{mL}, \quad 0.653 \mu \mathrm{g} / \mathrm{mL}, \quad 1.307 \mu \mathrm{g} / \mathrm{mL}, \quad 2.613 \mu \mathrm{g} / \mathrm{mL}$, $5.227 \mu \mathrm{g} / \mathrm{mL}$ ) were analyzed by HPLC. Standard curves were manufactured with the concentration of standard solutions as abscissa and the average peak area of three times as ordinate.

After injected three times, the average peak area of the sample was obtained from the liquid chromatogram and then the concentration of quercetin was figured out through the standard curve of quercetin and the substrate concentration.

\subsection{Taxonomical investigations of active strain}

The identification of bioactive strain was conducted based on 16S rDNA phylogenetic analysis and morphological observation, as well as determination of physiological and biochemical features.

The genomic DNA of tested strain was extracted with phenol-chloroform method (Marmur, 1961), 16S rDNA was amplified by polymerase chain reaction using universal forward primer $27 \mathrm{~F}$ (5'-AGAGTTTGATCMTGGCTCAG-3') and reverse primer 1525R (5'-AGAAAGGAGGTGWT CCARCC-3') (Lane, 1991) with described procedure (Lu et al., 2001). Purified PCR products were directly sequenced by Beijing Sunbiotech Corporation. The sequence obtained was initially estimated by the BLAST facility of NCBI (www.ncbi.nlm.nih.gov/BLAST) and then aligned with all related sequences obtained from GenBank by BioEdit (Hall, 1999). Evolutionary distance matrices were calculated by using the method of Kimura 2-parameter and a neighbour-joining tree was reconstructed by the Mega 5.1 program (Saitou \& Nei, 1987, Tamura et al., 2011).

Cell morphology was examined with a light microscopy (Olympus, BH-2). Presence of spore was examined by staining using Schaeffer-Fulton method, and Gram reaction was determined using the bioMe'rieux Gram Stain kit according to the manufacturer's instructions (Beijing Land Bridge Technology Co., Ltd). All other test methods were based on those of Gordon et al. (Gordon, 1973) and have been described previously (Priest, 1988).

\section{Results}

\subsection{Screening result of active strain}

From the soil samples, 80 bacteria strains were isolated first on the beef extract peptone medium plate at $25^{\circ} \mathrm{C}$. Three strains, B12, C20 and D3, with potential activity of transforming rutin to quercetin were obtained after TLC determination (Figure 2).

The conversion capabilities of the three strains were re-screened by HPLC with rutin and quercetin standards as control. Retained time of rutin was $5.277 \mathrm{~min}$, and retained time of quercetin was $19.944 \mathrm{~min}$ (Figure 3). The results suggested that rutin and quercetin could be separated effectively under the experiment condition. Among the three positive strains of preliminary screened, strain D3 had an evident elution peak corresponding to quercetin and showed the highest conversion ability (Figure 3). Thus strain D3 was selected for further analysis quantitatively.

After the cultivation of strain D3 in the rutin-Beef extract-peptone broth for $24 \mathrm{~h}$, both the fermentation supernatant and suspension of cell precipitation were prepared and checked conversion activity. The quercetin was only found in cell precipitation transfer system, which suggested that the active enzyme was intracellular enzyme.

Standard curves were manufactured by Excel2003 in the further quantitative study. The regression equation of rutin was $y=12002 x+946.85\left(R^{2}=0.9977\right)$, and the regression equation of quercetin was $y=26141 x+1812.8$ $\left(R^{2}=0.9974\right)$ (Figure 4 and Figure 5). The results showed that rutin and quercetin had good linearities within the tested concentration range. After injected three times, the average peak area of quercetin was 10767498 in D3 sample. According to the quercetin standard curve, quercetin concentration of D3 sample was $0.412 \mathrm{mg} / \mathrm{mL}$, and transformation rate was $41.6 \%$ when transferred by the $50 \mathrm{mg}$ wet cell for $72 \mathrm{hr}$ at $25^{\circ} \mathrm{C}$ in the system of $4 \mathrm{~mL}$ containing $0.1 \%$ rutin. 


\subsection{Identification result of strain D3}

Nearly complete $16 \mathrm{~S}$ rDNA sequence of $1501 \mathrm{bp}$ of D3 strain was obtained. When comparing the 16S rDNA sequence with the GenBank database, the greatest similarity of $99.47 \%$ was found between strain D3 and Paenibacillus glucanolyticus DSM5162 ${ }^{\mathrm{T}}$ (AB073189). In the neighbour-joining phylogenetic tree, strain D3 and Paenibacillus glucanolyticus $\mathrm{DSM} 5162^{\mathrm{T}}$ clustered together with a high bootstrap value of $100 \%$ (Fig.6).

Colonies of the strain D3 were flat, smooth, and opaque and were motile during growth on nutrient agar plates. Cells were 3.0 3.5 $\mu \mathrm{m} \times 0.8 \sim 0.9 \mu \mathrm{m}$, Grampositive, and produced oval terminal spores that markedly distend the sporangium. In physiological and biochemical experiments, strain D3 did not produce gas from glucose, and produced acid from cellobiose, D-fructose, D-galactose, D-glucose, lactose, maltose, D-mannose, sucrose, trehalose, D-xylose. In Voges-Proskauer broth the $\mathrm{pH}$ was 5.3. The strain hydrolyzed arbutin, DNA, pullulan, ribonucleic acid and starch, and did not hydrolyze allantoin, chitin, elastin, pectin, tyrosine, or urea. Strain D3 could grow in the presence of $5 \% \mathrm{NaCl}$ but not in the presence of $10 \% \mathrm{NaCl}$ and was negative for indole production and the Voges-Proskauer reaction. All these phenotype characteristics serve to classify strain D3 as a member of the species Paenibacillus glucanolyticus. There for this active strain was identified as Paenibacillus glucanolyticus $\mathrm{D} 3$ on species level.

\section{Discussion}

TLC method was widely used in separation and determination of flavonoids including rutin and quercetin (Zhou et al., 2006). In this research, we obtained three strains that had the potential activity of transforming rutin to quercetin. But when they were checked by HPLC, only strain D3 exhibited a strong activity. The result indicated that TLC method only fit for preliminary screening of active strains. Although the transformation rate of strain D3 from rutin to quercetin is not as high as the industrial production needed, this wild type bacterium will illustrate a good prospect of application after breeding and optimization of transformation conditions.

The active strain D3 of this research was identified to be Paenibacillus glucanolyticus, i.e. a bacillus, which is different from other strains that reported mainly fungi and actinobacteria. From another point of view, we found a new microbial resource for biotransformation of rutin to quercetin, and will offer a new technology for quercetin production.

Moreover, the rutin degrade enzyme of P. glucanolyticus D3 is produced constitutively, contrasting to the reported enzymes of Aspergillus, Penicillum, Thermoactinomyces and Streptococcus, which are induced rutin degrade enzymes of different types (Krishnamurty et al., 1970; Narikawa et al., 2000; Yang et al., 2009; Mamma et al., 2004). This characteristic enables P. glucanolyticus D3 with lower cost in enzyme production. However, the enzymology characteristics, conversion conditions and breeding of the higher active strain ect, further studies are still needed to be carried out.

\section{References}

Cheng, X., Chen, X., Gao, Y., et al. (2004). Determined the contents of rutin and quercetin by RP-HPLC. Chinese Traditional Patent Medicine, 26(8), 680-682.

Gordon, R.E., Haynes, W.C., \& Pang, C. (1973). The genus Bacillus. Agriculture Handbook no. 427. United States Department of Agriculture, Washington, D. C.

Hall, T.A. (1999). BioEdit, a user-friendly biological sequence alignment editor and analysis program for Windows 95/98/NT. Nucleic Acids Symp Ser, 41, 95-98.

Jia, D., Li, S., \& Yang, H. (2008). RP-HPLC Determination of rutin and isoquercitrin in mulberry leaves(Morus alba L.). Food Science, 29(8), 499-501.

Jiang, P., Burczynski, F., Campbell, C., et al. (2007). Rutin and flavonoid contents in three buckwheat species Fagopyrum esculentum, F. tataricum, and F. homotropicum and their protective effects against lipid peroxidation. Food Research International, 40(3), 356-364. http://dx.doi.org/10.1016/0022-0248(95)00133-6

Jin, Y., Lü, Y., Han, G., et al. (2007). Comparative study on in vitro anti-free radical activities of quercetin, isoquercetin, and rutin. Chinese Traditional Patent Medicine, 38(3), 408-412.

Jin, Y., Lü, Y., Han, G., et al. (2009). The study on structure activity relationship and protective effect in the hypoxia/hypoglycemia model of HEK293 cells of quercetin and Glucoside. Chinese Traditional Patent Medicine, 40(4), 618-620.

Kang, C., Wen, L., \& Ding, Z. (2000). Studies on separation and identification of flavonoid compositions with microemulsion thin layer chromatography. Chinese Journal of Pharmaceutical Analysis, 20(2), 121-123. 
Kimura, M. (1980). A simple method for estimating evolutionary rates of base substitutions through comparative studies of nucleotide sequences. Journal of Molecular Evolution, 16, 111-120. http://dx.doi.org/10.1007/BF01731581

Krishnamurty, H.G., \& Simpson, F.J. (1970). Degradation of rutin by Aspergilis flavus. The Journal of Biological Chemistry, 245(6), 1467-1471.

Lane, D.J. (1999). 16S/23S rDNA Sequencing. In: Nucleic acid techniques in bacterial systematics (eds Stackebrandt, E., Goodfellow, M.). John Wiley \& Sons, Chichester, pp115-175.

Lu, Z., Liu, Z., Wang, L., et al. (2001). Saccharopolyspora falva sp. nov. and Saccharopolyspora thermopohila sp.nov., novel actinomycetes from soil. International Journal of Systematic and Evolutionary Microbiology, 51, 319-325.

Macdonald, I.A. \& Bussard, R.G. (1984). Rutin indueed $\alpha$-Glucosidase activity in Streptococcus faeeium VGH-1 and Streptococcus sp. strain FRP- 17 isolated from human faces: formation of the mutagen, quercetin, from rutin. Applied and Environmental Microbiology, 25(1), 350-355

Mamma, D., Kalogeris, E., Hatzinikolaou, D. G., et al. (2004). Biochemical characterization of the multi-enzyme system produced by Penicillium decumbens grown on rutin. Food Biotechnology. 18(1), 1-18. http://dx.doi.org/10.1081/FBT-120030382

Marmur, J. (1961). A procedure for the isolation of deoxyribonucleic acid from microorganisms. Journal of Molecular Biology, 3, 208-218. http://dx.doi.org/10.1016/S0022-2836(61)80047-8

Narikawa, T., Shinoyama, H., \& Fujii, T. (2000). A $\beta$-rutinosidase from Penicillum rugulosum IFO 7242 that is a peculiar flavonoid glycosidase. Bioscience, Biotechonoly and Biochemistry, 64(6), 1317-1319. http://dx.doi.org/10.1271/bbb.64.1317

Priest, F, G., Goodfellow, M., \& Todd, C. (1988). A numerical classification of the genus Bacillus. Journal of Genetic Microbiology, 47, 1039-1046.

Saitou, N., \& Nei, M. (1987). The neighbor-joining method: a new method for reconstructing phylogenetic trees. Molecular Biology and Evolution, 4, 406-425.

$\mathrm{Su}$, J., \& Guo, C. (2002). Comparative study on the antioxidant capacity of quercetin in vivo and in vitro. Chinese Journal of Applied Physiology, 18(4), 382-386.

Sun, G., Zhao, Q., Dong, X., et al. (2009). Studies on the chemical components and pharmacological activities of Sophora japonica. Journal of Traditional Chinese Veternary Medicine.

Tamura, K., Peterson, D., Peterson, N., et al. (2011). MEGA5: Molecular evolutionary genetics analysis using maximum likelihood, evolutionary distance, and maximum parsimony methods. Molecular Biology and Evolution, 28, 2731-2739. http://dx.doi.org/10.1093/molbev/msr121

Yang, C.H., Huang, Y.C., \& Chen C.Y. (2009). Degradation of rutin by Thermoactinomyces vulgaris and other thermophilic compost isolates. Journal of Agricutral and Food Chemistry, 57, 5095-5099. http://dx.doi.org/10.1021/jf900617z

Zhou, X., Song, F., \& Zhong, Z. (2006). Determination of rutin and quercetin in Flos Sophorae with thin layer chromatogram scanner. Chinese Journal of Experimental Traditional Medical Formulae, 12(8), 14-16. 

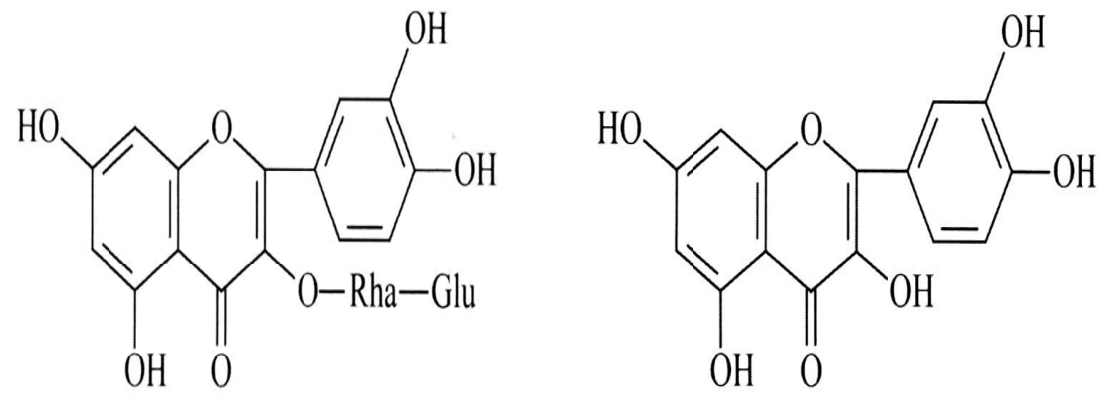

Figure 1. Chemical structures of rutin (left) and quercetin (right)

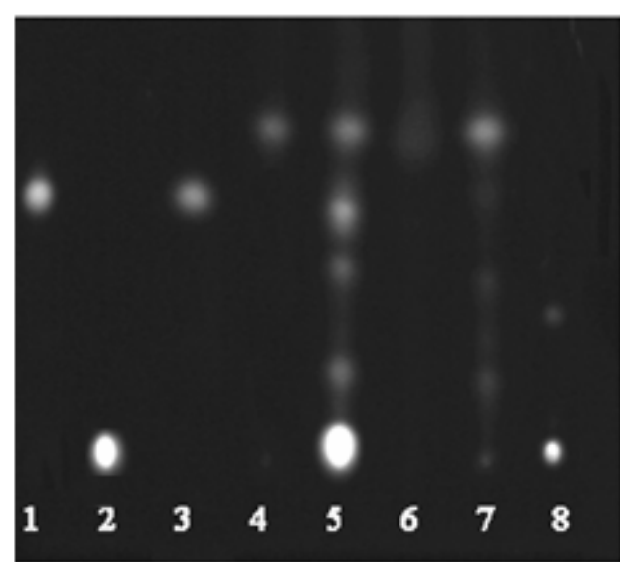

Figure 2. TLC determination result of some bacterial samples

1 rutin, 2 quercetin, 3 negative control, 4-8 samples (5. D3) 


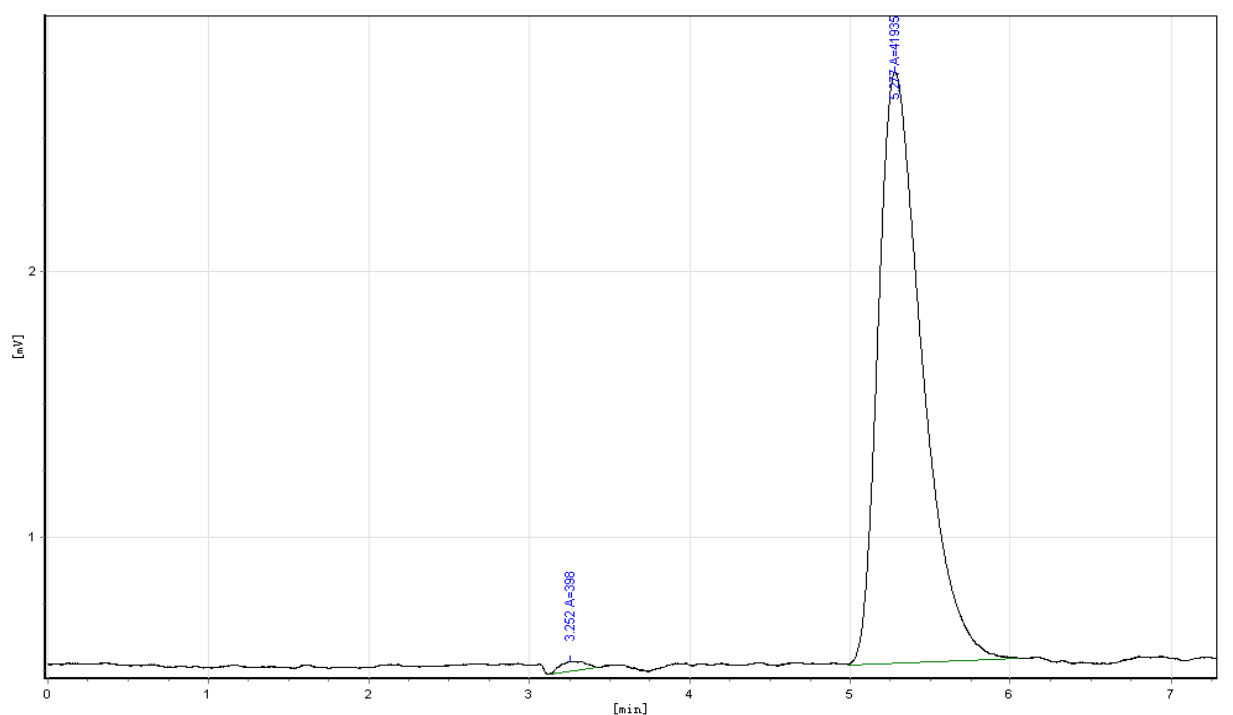

(a)

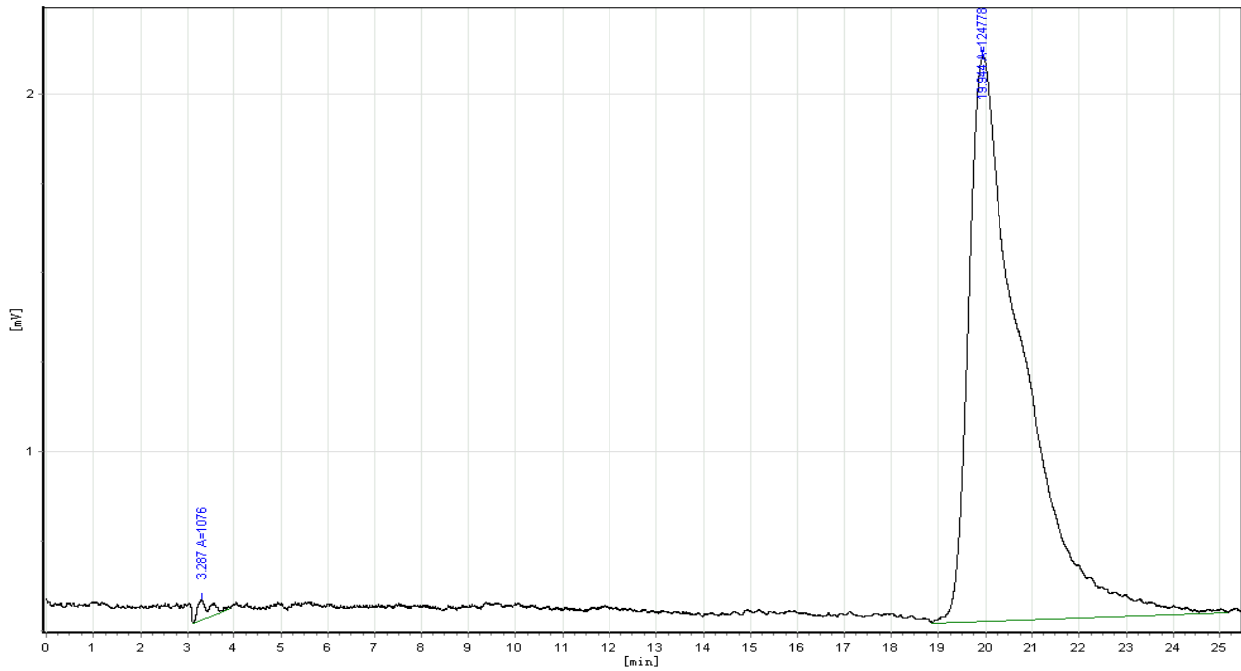

(b)

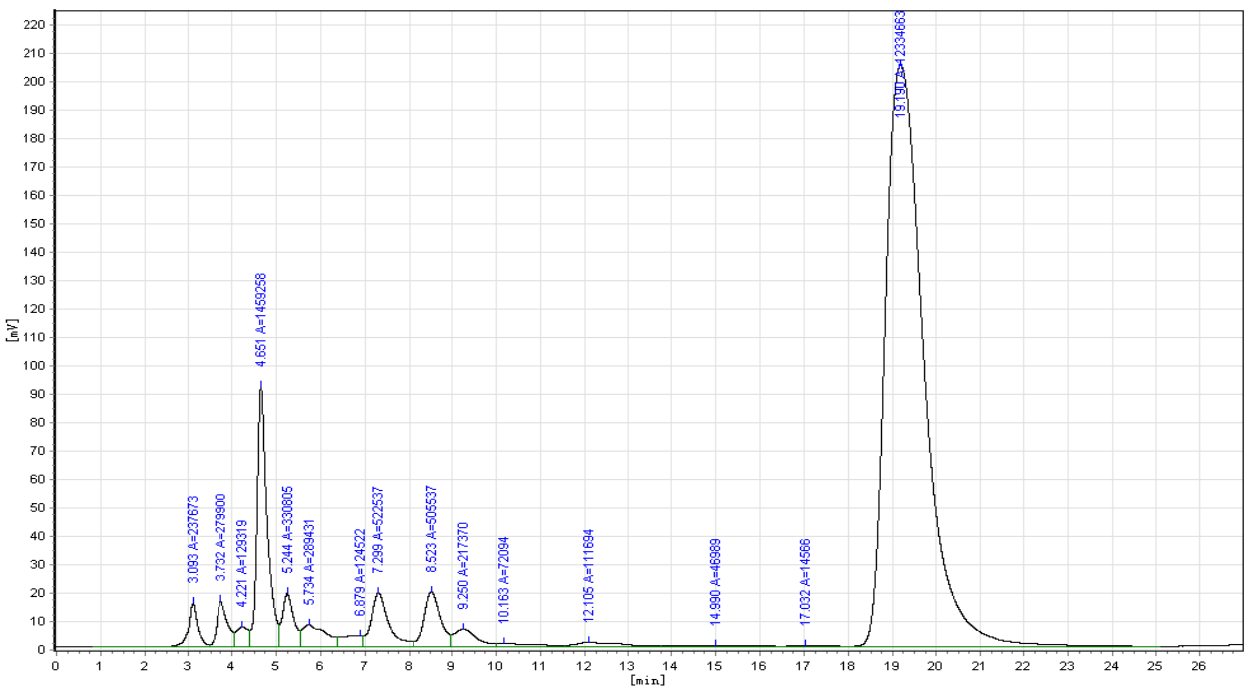

(c)

Figure 3. HPLC chromatograms of rutin standards and tested sample

(a) rutin standard, (b) quercetin standard, (c)tested sample D3 


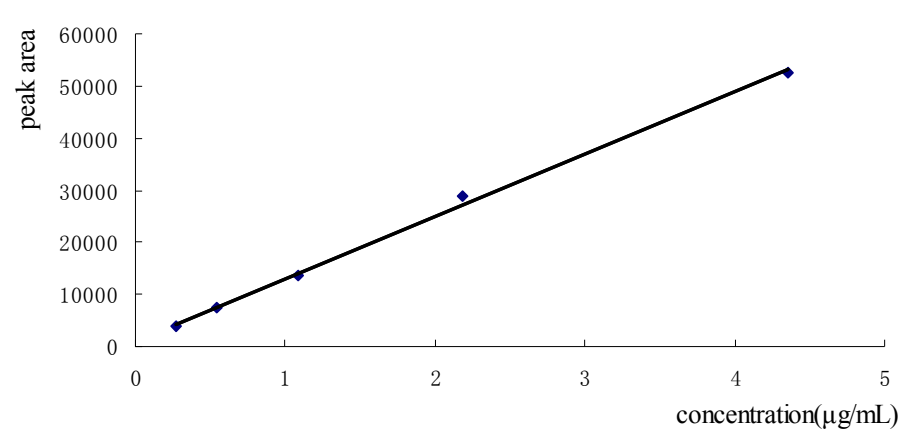

Figure 4. Standard curve of rutin by HPLC

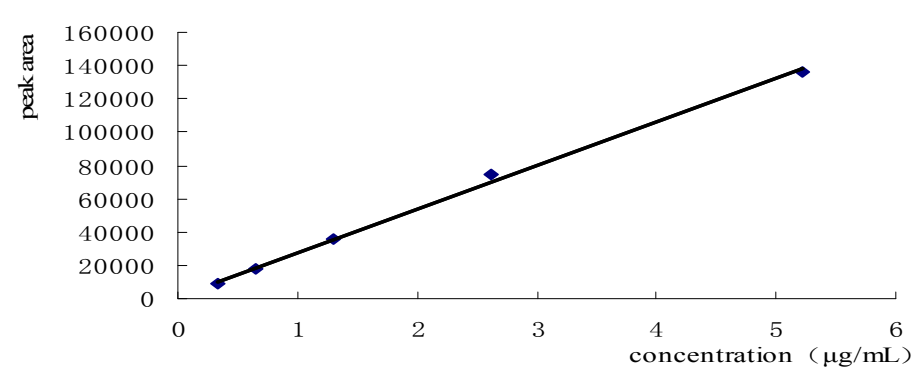

Figure 5. Standard curve of quercetin by HPLC

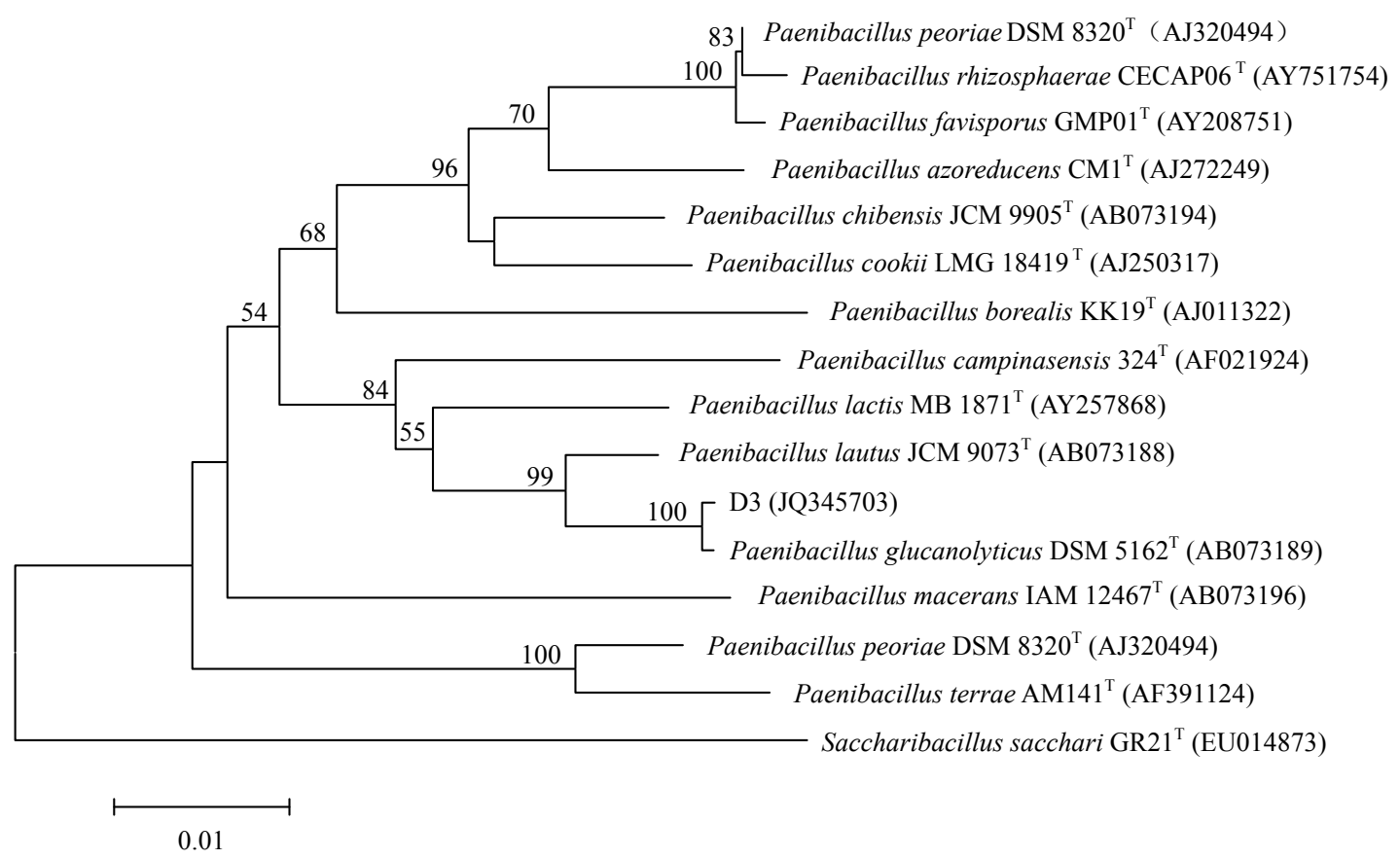

Figure 6. Neighbour-joining phylogenetic tree of strain D3 and related Paenibacillus representatives based on almost complete 16S rDNA sequences using Saccharibacillus sacchari (EU014873) as outgroup 